\title{
Eficiência Energética com Modelo de Grafo Auxiliar para Redes Ópticas Elásticas
}

\author{
Lucas R. Costa, André C. Drummond \\ Departamento de Ciência da Computação - Universidade de Brasília (UnB), \\ 70910-900, Brasília, Brasil \\ lucasrc.rodri@gmail.com, andred@unb.br
}

\begin{abstract}
The emergence of Elastic Optical Networks (EON) has brought new conceptions in the operations of optical networks, improving its flexibility and efficiency. The routing and spectrum assignment (RSA) is one of the key problems in EON and which deals with resource allocation. In order to reduce the bandwidth blocking rate on the network, recently, Auxiliary-Graph-Model approaches propose spectrum reservation schemes to reduce the network exhaustion probability. However these approaches do not take into account the energy expenditure caused by these schemes. In this work, we use an auxiliary graph model to design a new RSA heuristic algorithm to improve the network energy consumption without losses in bandwidth blocking rate. Numerical results show that our proposal may provide a reduction of up to $54 \%$ in the bandwidth blocking ratio and energy savings of up to $10 \%$ compared to literature.
\end{abstract}

Resumo. O surgimento das redes ópticas elásticas (EON) trouxe novas concepções nas operações das redes de núcleo, melhorando a flexibilidade da rede e sua eficiência no uso dos recursos. O roteamento e atribuição do espectro (Routing and Spectrum Assignment - RSA), que é responsável pela alocação de recursos, é um dos principais problemas das redes EON. Recentemente algumas abordagens baseadas em modelo de grafo auxiliar estão sendo propostas como soluções RSA. Estes modelos utilizam esquemas de reserva de espectro para reduzir a probabilidade de exaustão da rede. Embora essas abordagens proporcionem uma baixa probabilidade de bloqueio na rede, elas não levam em consideração o gasto energético provocado por esses esquemas. Este trabalho propõe uma nova abordagem RSA baseada em grafo auxiliar que melhora o consumo de energia da rede sem perdas na taxa de bloqueio de largura de banda. Os resultados numéricos mostram que a proposta apresentada pode fornecer uma redução de até $54 \%$ na taxa de bloqueio de banda e uma economia energética de até $10 \%$ em comparação com as abordagens da literatura.

\section{Introdução}

Reduzir o consumo energético de todos os processos da humanidade tornou-se uma meta obrigatória nos últimos anos devido ao esgotamento rápido das reservas de combustível fósseis e o aumento do custo da geração de energia [Dharmaweera et al. 2015]. O setor de Tecnologia da Informação e Comunicação (TIC) vem ganhando destaque entre os consumidores globais de energia. Com base nas estimativas de [Andrae and Corcoran 2013], o consumo de energia das TICs representou aproximadamente $4 \%$ a $7,4 \%$ do consumo 
global de energia em 2009 a 2012. As previsões, no pior dos casos, mostram um aumento de $12 \%$ em 2017, impulsionado principalmente pela expansão da infraestrutura das redes de núcleo e dos centros de dados [Andrae and Corcoran 2013].

As redes de telecomunicações estão entre as que mais consomem energia dentro do setor das TICs. Seu custo é cerca de $37 \%$ do consumo de energia total da área e alguns dados surpreendentes reforçam ainda mais esta estimativa. Por exemplo, em 2012, o setor de telecomunicações por si só consumiu mais de 250 Terawatt-hora (TWh) de eletricidade no mundo [Dharmaweera et al. 2015]. Uma das principais razões para esse aumento no gasto de energia foi o crescimento do tráfego na Internet. Nos últimos anos, o tráfego da Internet vem crescendo exponencialmente devido as aplicações emergentes, tais como TV de alta definição, computação em nuvem, aplicações multimídia e sistemas em tempo real. O tráfego nas redes de núcleo tem crescido $24 \%$ por ano em média desde 2016 , de acordo com [Cisco 2017]. Este contínuo aumento no tráfego da Internet fez com que a academia e a indústria concentrassem seus esforços no aumento da capacidade da rede, deixando de lado alguns fatores como o consumo energético.

O aumento na capacidade da rede normalmente é acompanhado por um aumento no consumo de energia do equipamento de rede [Vizcaíno et al. 2012]. Dessa forma, o crescimento do tráfego torna a questão do consumo de energia um problema significativo. Este aumento no consumo de energia não tem apenas relações econômicas, mas também consequências ecológicas. De acordo com [Idzikowski et al. 2016], o setor de telecomunicações por si só é responsável por $2 \%$ de emissões globais de dióxido de carbono. Tendo em vista que o impacto das TICs no consumo de energia mundial é não negligenciável, a academia e a indústria têm investido em pesquisas para aumentar a eficiência energética nos seus recursos, principalmente no que tange às redes de núcleo [Idzikowski et al. 2016].

Neste cenário, a literatura vem buscando soluções baseadas em redes conscientes do consumo energético, denominadas Energy-Aware (EA) Networks [Vizcaíno et al. 2012]. Uma rede com custo eficiente e com eficiência energética exige uma tecnologia capaz de aproveitar melhor os recursos disponíveis na rede e reduzir o hiato entre a utilização da rede e a capacidade oferecida [Nag et al. 2013]. A literatura divide as abordagens EA em duas categorias, denominadas: (i) Proporcional à Energia (Energy-proportional); e (ii) Modo de Suspensão (Sleep mode) [Idzikowski et al. 2016]. As propostas baseadas na primeira abordagem procuram relacionar o gasto energético do dispositivo com sua capacidade e carga trafegada. Neste contexto, a energia consumida do dispositivo é proporcional ao uso do equipamento, dispositivos que trafegam mais dados gastam mais energia. A segunda abordagem afeta a rede como um todo distribuindo cuidadosamente a carga de tráfego da rede em poucos equipamentos, de modo que, alguns dispositivos são totalmente utilizados e outros dispositivos ficam ociosos e são colocados em modo de suspensão [Dharmaweera et al. 2015].

De acordo com trabalhos recentes, as abordagens Sleep mode são motivadas pelo fato que o consumo de energia dos dispositivos atuais é praticamente independente da carga. Assim, o uso de menos dispositivos é mais eficiente em termos energéticos do que o uso de muitos dispositivos subutilizados (Energy-proportional) [Heddeghem et al. 2012, Idzikowski et al. 2016]. 
As EONs vem atraindo um grande interesse pela academia devido a perspectiva de gerenciamento ágil do espectro óptico e a flexibilidade na alocação de recursos. Seu surgimento trouxe novas concepções nas operações das redes ópticas, melhorando a flexibilidade da rede e sua eficiência [Tomkos et al. 2014]. Em comparação com as redes ópticas tradicionais de multiplexação por divisão de comprimento de onda (Wavelength-Division Multiplexing - WDM), as EONs implementam mecanismos de alocação de largura de banda mais sofisticados, (i) baseados em subportadores ópticas OFDM (Orthogonal Frequency-Division Multiplexing), (ii) em abordagens de roteamento e de atribuição do espectro (Routing and Spectrum Assignment - RSA) e (iii) transmissores e comutadores ópticos de largura de banda variável (Bandwidth-Variable transponder - BVT e Bandwidth-Variable Optical Cross-Connects - BV-OXC) [Tomkos et al. 2014].

O problema de roteamento e atribuição de espectro (RSA) é um dos principais problemas no EON. Seu objetivo é lidar com o gerenciamento de recursos ao encontrar um caminho e atribuir um espectro contínuo e contíguo para cada solicitação de conexão. Abordagens de agregação de tráfego elétrico (Electrical Grooming - EG) e ópticos (Optical Grooming - OG) foram recentemente propostas em redes EON. Essas abordagens têm sido utilizadas para melhorar o consumo de energia da rede reduzindo o número de caminhos ópticos e desligando o maior número possível de dispositivos na rede [Dharmaweera et al. 2015].

Recentemente, algumas abordagens baseadas em Grafo Auxiliar (GA) foram utilizadas para resolver o problema RSA com agregação de tráfego em EON [Zhang et al. 2013, Zhang et al. 2015]. Essas soluções são capazes de reduzir a taxa de bloqueio de banda na rede através de esquemas de reserva de espectro na alocação de recursos, contribuindo para a redução de sua probabilidade de exaustão. Embora as redes de núcleo sejam dimensionadas para demandas de tráfego elevadas, a quantidade de dados que flui na rede está normalmente abaixo das taxas de dados máximas suportada [Idzikowski et al. 2016]. Tais abordagens não levam em consideração o gasto de energia proporcionado por esses esquemas de reserva de espectro. O uso dessas políticas acarreta um aumento no consumo de energia dos dispositivos da rede.

Com base no fato de que os dispositivos de rede consomem uma determinada quantidade de energia independentemente da quantidade de dados processados [Idzikowski et al. 2016], este trabalho propõe uma nova solução RSA baseada em uma modelagem de grafo auxiliar com agregação de tráfego elétrico (EG) e óptico (OG). A proposta tem como objetivo melhorar o consumo de energia da rede através do uso de abordagens Sleep mode sem prejudicar o desempenho na taxa de bloqueio de banda da rede. Os resultados numéricos mostram que a proposta apresentada é capaz de obter um desempenho melhor na taxa de bloqueio em comparação com as abordagens da literatura, reduzindo os gastos de energia na rede em até $10 \%$.

\section{Trabalhos Relacionados}

A seguir, na Seção 2.1, são apresentados alguns trabalhos relacionados às abordagens baseadas em grafo auxiliar utilizadas para alocação de espectro em redes ópticas. Em seguida, na Seção 2.2, são detalhados alguns trabalhos relacionados à eficiência energética das redes de núcleo. 


\subsection{Modelos de Grafo Auxiliar}

Os grafos são comumente usados para modelar problemas de alocação de recursos, especialmente em redes ópticas. Os autores em [Zhu et al. 2003] propõem um modelo de grafo auxiliar para o problema RWA (Routing and Wavelength Assignment) com agregação de tráfego para redes WDM. A proposta baseia-se em um modelo que atinge vários objetivos através de diferentes políticas de agregação por meio da manipulação das arestas no grafo auxiliar. Vários esquemas de seleção de tráfego baseados nesta abordagem são propostos e seus desempenhos foram avaliados para diferentes topologias de rede.

Baseados na ideia de [Zhu et al. 2003], os autores em [Zhang et al. 2013] investigam o problema da agregação elétrica para redes EON. Os autores propõem o uso de um grafo auxiliar para a implementação de diversas políticas de agregação de tráfego. Uma reserva de espectro para a agregação de futuras demandas de tráfego também é proposta. A ideia por trás deste esquema é ampliar o tamanho do canal, criado pelo BVT, para a acomodação de futuras requisições de tráfego através da agregação elétrica (EG). Os resultados mostraram que existem diferentes compromissos entre as políticas e estas devem ser adotadas de acordo com os objetivos e as circunstâncias da rede.

Baseados em [Zhang et al. 2013], os autores em [Zhang et al. 2015] propõem o uso de um grafo auxiliar para resolver o problema RSA com técnicas de agregação de tráfego. Eles introduzem o uso da agregação óptica no grafo auxiliar. Três políticas de agregação de tráfego são propostas por meio do ajuste nos pesos das arestas do grafo. A primeira política baseia-se na minimização do número de caminhos ópticos estabelecidos. Duas abordagens foram criadas para atender essa política a MEG (Maximal Electrical Grooming) e a MOG (Maximal Optical Grooming) ambas procuram realizar o máximo de agregação elétrica e óptica, respectivamente. A segunda política tem como objetivo reduzir o número de saltos virtuais da rede (Minimal number of Virtual Hops - MVH). Essa abordagem causa a redução no número de conversões eletro-óptica na rede ao custo de estabelecer caminhos ópticos mais longos. A terceira e última política leva em consideração a diminuição do número de saltos físicos na rede (Minimal number of Physical Hops - MPH). Essa abordagem causa o estabelecimento de caminhos ópticos menores e um menor número de dispositivos BV-OXCs utilizados na rede. Além disso, os autores propõem uma nova política de reserva de espectro. O novo esquema de reserva, denominado SRNP (Spectrum Reservation for each Node-Pair), procura realizar reservas de espectro entre pares de nós. Diferentemente do esquema proposto em [Zhang et al. 2013], onde o SRLP (Spectrum Reservation for each LightPath) cria uma reserva de espectro para cada caminho óptico estabelecido. Os resultados também mostraram que existe uma compensação entre as diferentes políticas de agregação de tráfego e estas devem ser adotadas de acordo com os objetivos e as circunstâncias da rede dinâmica.

Embora ambas as ideias de reserva do espectro de [Zhang et al. 2013] e de [Zhang et al. 2015] reduzam a probabilidade de exaustão da rede, esses trabalhos não medem a eficiência energética. Essas abordagens utilizam uma quantidade maior de recursos da rede o que acarretará em um consumo maior de energia na rede, como será apresentado na Seção 4.

\subsection{Eficiência Energética}

A literatura vem estudando diferentes soluções para redução do desperdício energético ou, de forma equivalente, para tornar o consumo da rede proporcional à carga do tráfego. 
Em [Vizcaíno et al. 2012], os autores avaliam a eficiência energética em redes WDM e EON no cenário de tráfego dinâmico. Vários métodos são apresentados para mensuração dos gastos energéticos dos diferentes componentes das arquiteturas WDM e EON. Algoritmos heurísticos para alocação de espectro em ambos os cenários de rede, com foco na economia energética, são desenvolvidos. As heurísticas no cenário EON levam em consideração o problema de roteamento, modulação e atribuição de espectro (Routing Modulation Level and Spectrum Assignment - RMLSA) que inclui a atribuição do formato de modulação do espectro, considerando a distância de transmissão, no problema RSA. Conclui-se que a rede EON possui a solução mais eficiente em termos energéticos, uma vez que é capaz de lidar com uma maior quantidade de tráfego utilizando menos recursos.

Os autores em [Nag et al. 2013] comparam a eficiência energética de redes EON e redes MLR (Mixed-Line-Rate) para o cenário de tráfego estático. São propostos modelos de MILP (Programação Linear Inteira Mista) para ambas as redes com o objetivo de modelar a eficiência energética em dois cenários. Os resultados mostraram que a rede EON é superior em termos de eficiência energética, especialmente quando o tráfego na rede leva picos aleatórios em alguns dos enlaces.

Em [Wu et al. 2014] os autores propõem o primeiro estudo sobre eficiência energética utilizando técnicas de agregação de tráfego EG e OG para redes EON. Os autores formulam um modelo de ILP (Programação Linear Inteira) baseado nas técnicas de agregação com o objetivo de minimizar o gasto energético. Os autores também desenvolvem uma heurística e duas políticas de alocação de espetro: $(i)$ com foco no gasto espectral, denominada GGA-MS (Green Grooming Algorithm with Minimal Spectrum Consumption); (ii) com foco no gasto energético, denominada GGA-MP (Green Grooming Algorithm with Minimal Power Consumption). Os resultados de simulação mostram a eficiência da heurística proposta em termos da proximidade com os resultados da PLI.

\section{Solução Proposta}

Baseando-se na literatura de EA, este trabalho propõe um novo algoritmo RSA, baseado em um modelo de grafo auxiliar (AG) inovador, com o objetivo de melhorar o consumo de energia da rede através da abordagem Sleep mode. Baseados nos modelos de grafo auxiliar de [Zhu et al. 2003] para redes WDM, os de [Zhang et al. 2013, Zhang et al. 2015] e o de [Wu et al. 2014] para redes EON, foi desenvolvido um novo modelo de grafo auxiliar que combina essas propostas usando técnicas de agregação elétrica e óptica que visam utilizar uma menor quantidade de dispositivos (BVT e BV-OXC) conectados na rede.

A topologia da rede é definida como $G=(V, E)$, onde $V$ é o conjunto de nós e $E$ é o conjunto de enlaces bidirecionais da rede. No cenário de tráfego dinâmico, os pedidos de conexão chegam um a um, mantêm-se por um certo período (holding-time) na rede e depois partem. O conjunto de requisições de conexão $R=\left\{r_{1}, r_{2}, \ldots, r_{n}\right\}$ é definido. Cada pedido de conexão é representado por $r_{i}(s, d, b)$, onde $s$ é o nó de origem e $d$ é o nó de destino da requisição, $b$ é a largura de banda do pedido de conexão.

Quando uma requisição $r_{i}$ chega, o plano de controle determina como provisionála determinando sua rota através de caminhos ópticos existentes ou novos. O uso de um caminho óptico existente levará ao uso da agregação de tráfego elétrica. O uso de um novo caminho óptico pode ocorrer em duas situações: $(i)$ usando um transmissor (BVT) 
existente (agregação óptica); ou (ii) usando um novo transmissor e receptor para criação de um novo circuito óptico. A próxima seção mostra como o grafo auxiliar proposto coordena todas essas possibilidades.

\subsection{Grafo Auxiliar (AG)}

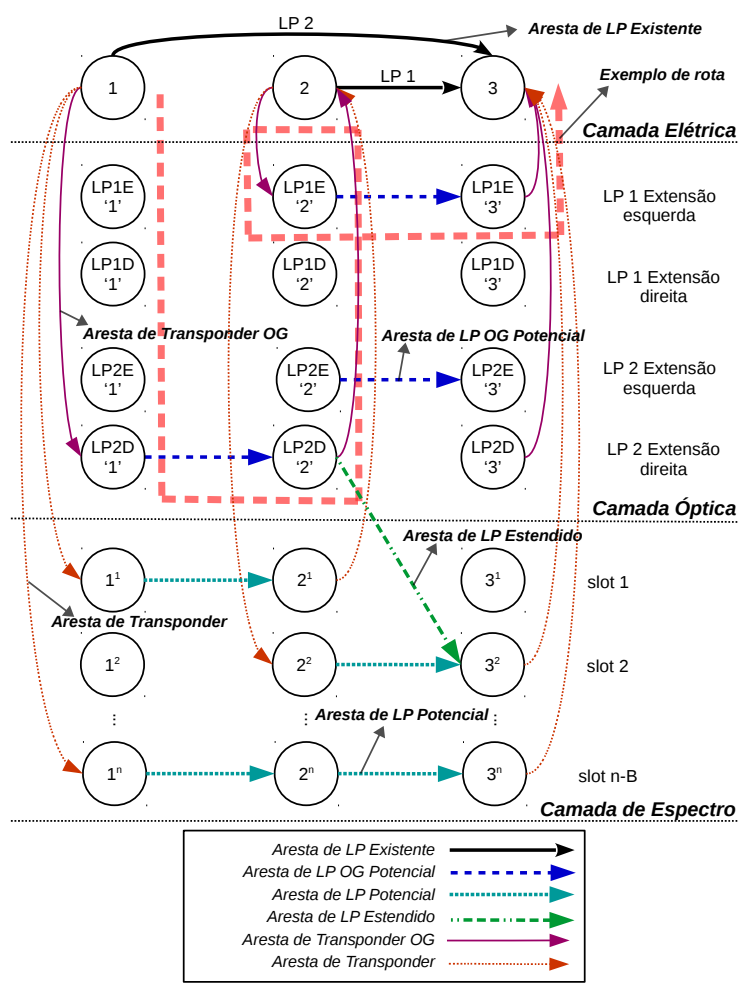

Figura 1. Grafo Auxiliar estabelecendo uma nova conexão entre os nós 1 e 3.

Da mesma forma que em [Zhang et al. 2015], o grafo auxiliar proposto resolve o problema RSA com agregação de tráfego. Como em [Zhang et al. 2015], a proposta também introduz o uso da agregação óptica no grafo auxiliar, porém de uma forma inovadora. A proposta deste trabalho soluciona o problema RSA com agregação de tráfego elétrico e óptico satisfazendo todas as restrições de continuidade e contiguidade do espectro em uma única solução.

O grafo AG de [Zhang et al. 2015] representa parcialmente o espectro, de maneira que cada uma de suas arestas corresponde a uma solução RSA independente de solução apresentada. Em contrapartida, neste trabalho, o espectro é amplamente representado através das inúmeras subcamadas do grafo e suas arestas, de tal forma que o AG proposto traz uma melhor representação do estado da rede. Isso permite a implementação de soluções de engenharia de tráfego mais elaboradas.

A Figura 1 mostra um exemplo de AG para uma topologia física linear de três nós (1,2 e 3) representando o estado da rede. O modelo de grafo auxiliar proposto é composto por três camadas: (i) A Camada Elétrica; (ii) a Camada Óptica; e (iii) a Camada de Espectro. Como em [Zhang et al. 2013] e em [Zhang et al. 2015], um novo grafo auxiliar é construído sempre que uma nova solicitação de tráfego chega. Os nós em cada camada do grafo auxiliar correspondem aos nós na topologia física. 
A Camada Elétrica representa a topologia virtual de interesse, considerando apenas os caminhos ópticos estabelecidos na rede que possuem capacidade suficientes para acomodar a chamada através de agregação elétrica. A Camada Óptica representa os caminhos ópticos estabelecidos que podem ser estendidos através da agregação óptica. Cada caminho óptico pode ser estendido para ambos os lados do espectro, esquerdo e direito, de modo que, para cada caminho óptico, existem pelo menos duas subcamadas na camada óptica que representam as possibilidades de agregação óptica neste caminho óptico. (por exemplo, LP 1 Extensão esquerda ou direita). Por último, a Camada de Espectro é usada para representar os recursos de espectro que podem ser utilizados para a composição de novos caminhos ópticos na rede. Para o estabelecimento de um novo caminho óptico, deve existir pelo menos $B+2 * G$ subportadoras (slots) OFDM contíguas não utilizadas em cada fibra óptica do caminho óptico, onde $B$ é a largura de banda requerida da conexão e $G$ é o tamanho da banda de guarda necessária, em slots. Além disso, devido à restrição de continuidade, a faixa de espectro do novo caminho óptico deve ser contínuo ao longo de todas as fibras do caminho. Portanto, há pelo menos $n-B$ subcamadas, onde $n$ é igual ao capacidade máxima de slots da fibra óptica.

Para conectar os nós na mesma camada, existem três tipos de arestas direcionais no AG, conforme descritas a seguir:

Aresta de LP Existente - ocorre caso haja um caminho óptico já estabelecido na rede e sua capacidade for suficiente para acomodar a banda da nova requisição de tráfego por meio da agregação elétrica. Caso haja mais de um caminho óptico estabelecido ligando os mesmos nós, o caminho óptico com maior capacidade será o escolhido.

Aresta de LP OG Potencial - indica que um caminho óptico pode ser estendido através da agregação óptica para o lado esquerdo ou direito do espectro no túnel óptico.

Aresta de LP Potencial - indica que existe recurso espectral contíguo e contínuo entre dois nós da camada física que atende a demanda de tráfego requerida $(B+2 * G)$ e, portanto, é possível estabelecer um caminho óptico por esses nós.

Para conectar os nós em camadas diferentes, existem três tipos de arestas direcionais no AG, conforme descritas a seguir:

Aresta de LP Estendido - ocorre se um caminho óptico pode ser estendido através da agregação óptica e possa ser estabelecido um novo caminho óptico através da bifurcação do túnel óptico.

Aresta de Transponder OG - representa a possibilidade de reutilização de um BVT por meio da agregação óptica. Para isso, é necessário atender restrições como capacidade máxima de subportadoras transmitidas e número de subtransmissores por BVT.

Aresta de Transponder - representa se existem BVTs livres para realização de uma conversão elétrica-ótica (E/O) ou óptica-elétrica (O/E) ainda não utilizada.

Um exemplo de rota para um pedido de conexão com origem no nó 1 e destino no nó 3 é apresentado na Figura 1. Neste exemplo existem pelo menos dois caminhos ópticos (LP 1 e LP 2) já estabelecidos na rede. A demanda é atendida através do roteamento multihop, utilizando dois caminhos ópticos para a transmissão e realizando uma conversão O-E-O no nó 2. Para o caminho do nó 1 ao 2, a conexão é realizada através da agregação óptica estendendo a frequência do espetro para direita no "LP 2". Para o caminho do nó 
2 ao 3, a conexão é realizada por meio da agregação óptica estendendo o espectro para a esquerda no "LP 1". Observe-se que não é possível estender a frequência do espectro para a esquerda no 'LP 2' na rota 1-2, o mesmo acontece para a direita na rota 2-3 no "LP 1". Outros exemplos de rotas poderiam ser encontrados no grafo apresentado. Por exemplo, a demanda poderia ser atendida através da agregação elétrica sob o "LP 2". Outra possibilidade seria a criação de um novo caminho óptico na subportadora $n$.

Algumas considerações sobre o grafo devem ser observadas. Qualquer agregação óptica deve ser realizada apenas no nó de origem do caminho óptico considerado (LP candidato). Por este motivo existe apenas uma Aresta de Transponder OG em cada subcamada na Camada Óptica. Tendo em vista que as subcamadas da Camada Óptica são formadas a partir dos caminhos ópticos já estabelecidos na rede, para evitar a alta complexidade de sua construção, executa-se (offline) o algoritmo de YenKSP entre os nós de origem e destino da requisição e são considerados apenas os caminhos ópticos dos nós pertencentes aos $k$ - menores caminhos. Isso limita o tamanho do grafo e evita uma maior complexidade na construção e na busca da solução proposta.

A principal contribuição desta proposta, em comparação com a literatura, é a forma como é construída a Camada Óptica. Sua construção possibilita um número maior de soluções RSAs com agregação óptica, pois cada aresta representa um conjunto de espectro viável que atende todas as restrições do problema RSA e, portanto, fornecendo uma solução RSA completa. Ao contrário do que é proposto em [Zhang et al. 2015], onde suas arestas são criadas a partir do algoritmo RSA $K$-Shortest-Path (KSP) com First Fit (FF) o que exige uma solução RSA auxiliar para seu grafo. Por este motivo o número de soluções RSA de [Zhang et al. 2015] é limitado e seu grafo restringe a representação do espectro EON quando comparado com o grafo auxiliar deste trabalho.

\subsection{Algoritmo RSA Dinâmico}

O Algoritmo RSA Dinâmico que manipula o grafo auxiliar proposto é descrito no Algoritmo 1. Para cada $r_{i} \in R$, o AG é construído de acordo com o estado atual da rede (linha 2 ). Todas as arestas que aparecem no AG devem satisfazer o requisito de largura de banda de $r$. Então, pode-se atribuir pesos das arestas de acordo com as diferentes Políticas de Eficiência Energética (Seção 3.3). Em seguida, executa-se o algoritmo de Dijkstra entre os dois nós da Camada Elétrica que correspondem à fonte $s$ e ao destino $d$ da requisição $r_{i}$ no grafo auxiliar (linha 3). A rota selecionada determina se é possível e como deve ser provisionado a requisição de tráfego $r_{i}$. Caso o algoritmo de Dijkstra não encontre um caminho, bloqueia-se a requisição (linha 5). Caso contrário, são estabelecidos novos caminhos ópticos e/ou são utilizados caminhos ópticos existentes, através da agregação de tráfego EG e OG (linha 7). Nota-se que o grafo auxiliar possibilita a utilização de mais de um caminho óptico para o atendimento da requisição, ou seja, permite o roteamento multihop através de conversões Óptico-Eletro-Óptico (OEO) na rede.

A complexidade do tempo da solução proposta é analisada da seguinte forma. Para a construção do $\mathrm{AG},|E| *|S|$ é número máximo de caminhos ópticos existentes na rede, onde $S$ é o conjunto de slots em cada enlace. Assim, a complexidade de tempo para a construção da Camada Elétrica é $O(|V|+|E| *|S|)$. Analogamente, o número máximo de subcamadas na Camada Óptica é $2(|E| *|S|)$, por causa da extensão para os dois lados do espectro, esquerda e direita, em cada caminho óptico. Dessa forma, a complexidade 


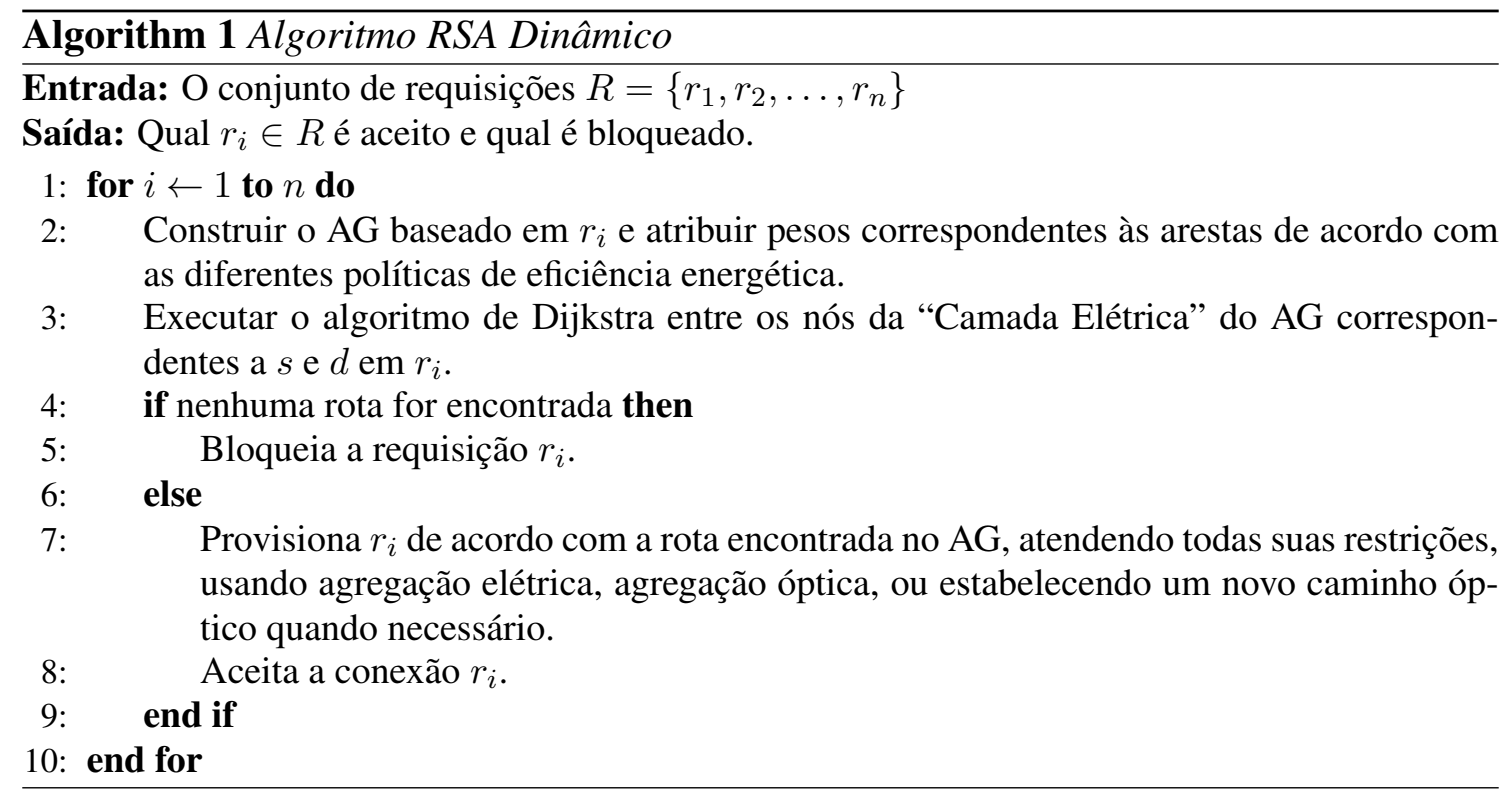

de tempo para a construção da Camada Óptica é $O\left((|V| * 2(|E| *|S|))^{2}\right)$ pois cada nó pode ter uma Aresta de LP OG Potencial e/ou uma Aresta de LP Estendido. O número de subcamadas da Camada de Espectro é $|S|$, assim a complexidade de tempo para sua construção com todas as Arestas de LP Potencial é $O\left((|V| *|S|)^{2}\right)$. O número máximo de Arestas de Transponder OG mais as Arestas de Transponder é $4 *(|E| *|S|)+2 *|S|$, assim a complexidade de tempo para construir todas as arestas é $O(4 *(|E| *|S|)+$ $2 *|S|)$. Finalmente, a complexidade de tempo para construção do AG é $O\left((|E||S|)^{2}+\right.$ $\left.\left(|V|^{2}\right)\right)$. Além disso, o número máximo de nós no grafo AG é $|V|+(2|E||S|)+(|V||S|)$, tomando isto igual a $M$, a complexidade de tempo para executar o Dijkstra no AG é $O(|M| \log |M|)$.

\subsection{Políticas de Eficiência Energética}

Com o modelo AG proposto (Seção 3.1), diversas políticas para a alocação de tráfego podem ser facilmente implementadas para atender às necessidades do operador da rede. Com o objetivo de melhorar o consumo de energia da EON, de acordo com a abordagem Sleep mode, este trabalho propõe duas políticas que proporcionam eficiência energética. A primeira é baseada na menor utilização de BVTs na rede, a segunda é baseada na minimização de BV-OXCs utilizados. Para a implementação dessas políticas, basta o ajuste nos pesos das arestas do grafo auxiliar proposto e a execução do Algoritmo 1.

A primeira política, chamada MinBVT, procura usar a capacidade máxima dos BVTs conectados através das técnicas de agregação de tráfego. Para isso, o AG é configurado de tal forma que o número de saltos na topologia virtual seja minimizado reduzindo o número de conversões OEO, como acontece nas políticas de [Zhang et al. 2013] e de [Zhang et al. 2015]. A política MinBVT aplica pesos as Arestas de Transponder OG e as Arestas de Transponder muito superiores a das demais arestas, de modo que a rota fornecida pelo algoritmo de Dijkstra evite o uso dessas arestas no AG, o que evitará o uso excessivo de BVTs. A segunda política chama-se MinBV-OXC e seu objetivo é minimizar o número de BV-OXCs utilizados pela requisição de tráfego. Para isso, pondera-se as arestas do AG de tal forma a minimizar o número de saltos físicos, 
como em [Zhang et al. 2013] e [Zhang et al. 2015]. Os pesos aplicados nas Arestas de LP Existente, Arestas de LP OG Potencial, Arestas de LP Potencial e Arestas de LP Estendido são muito maiores que os das demais arestas. Isso provocará rotas mais curtas no AG, portanto, uma requisição de tráfego será atendida com poucos saltos na topologia física e, consequentemente, menos BV-OXCs serão utilizados. Para a implementação destas políticas, os pesos das arestas são atribuídos de acordo com a Tabela 1.

Tabela 1. Atribuição dos pesos do AG para as políticas de eficiência energética

\begin{tabular}{|l|r|r|}
\cline { 2 - 3 } \multicolumn{1}{c|}{} & MinBVT & MinBV-OXC \\
\hline Aresta de LP Existente & 1,0 & 10 \\
\hline Aresta de LP OG Potencial & 0,01 & 10 \\
\hline Aresta de LP Pontencial & 0,01 & 10 \\
\hline Aresta de LP Estendido & 0,01 & 10 \\
\hline Aresta de Transponder OG & 50 & 0,1 \\
\hline Aresta de Transponder & 50 & 0,1 \\
\hline
\end{tabular}

\section{Avaliação de Desempenho}

Foram realizadas simulações para avaliar o desempenho do AG proposto (com o uso das políticas MinBVT e MinBV-OXC) em comparação com as propostas de [Zhang et al. 2013, Zhang et al. 2015] e [Wu et al. 2014] para resolver o problema RSA. Foram implementados os melhores algoritmos, em termos de desempenho da taxa de bloqueio de cada proposta da literatura. Dos algoritmos implementados em [Zhang et al. 2013], foi utilizado o LBSR (Load Balancing with Spectrum Reservation Schema). Das propostas implementadas em [Zhang et al. 2015], foram utilizadas o MPH (Minimal number of Physical Hops) e o MVH (Minimal number of Virtual Hops) com os dois esquemas de reserva de espectro proposto pelos autores: (i) SRLP (For Each Lightpath); e (ii) SRNP (For Each Node-Pair). Por fim, das propostas de [Wu et al. 2014], foi utilizado o algoritmo GGA-MP (Green Grooming Algorithm with Minimal spectrum Power Consumption).

\subsection{Ambiente de Simulação}

As simulações foram realizadas pelo simulador de rede ONS (Optical Network Simulator) [Costa et al. 2016]. Cada simulação foi realizada cinco vezes utilizando o método de replicações independentes. Para os resultados apresentados foram calculados intervalos de confiança com $95 \%$ de confiabilidade. Em cada simulação foram geradas $10^{5}$ requisições de conexão distribuídas uniformemente em 6 níveis de granularidade variando de $25 \mathrm{~Gb} / \mathrm{s}$ até $400 \mathrm{~Gb} / \mathrm{s}$ com passos de $25 \mathrm{~Gb} / \mathrm{s}$. O processo de chegada das chamadas segue a distribuição de Poisson com origem e destino distribuídos uniformemente para todos os pares de comunicação da rede. As topologias consideradas nas simulações foram a USANet com 24 nós e 43 enlaces bidirecionais, e a topologia PanEuro com 27 nós e 81 enlaces bidirecionais (Figura 2). Foi considerada a largura de banda de cada subportadora (slot) como sendo $12,5 \mathrm{GHz}$ e foi assumido que cada enlace possui a capacidade de 320 slots (4 THz). Admite-se uma banda de guarda de 2 slots $(25 \mathrm{GHz})$. Assume-se que cada nó na topologia possui 15 transmissores e cada transmissor tem a capacidade máxima de transmitir até 20 slots. Cada subportadora é capaz de transportar até $25 \mathrm{Gbps}$ por meio do formato de modulação QPSK (Quadrature Phase-Shift Keying). 

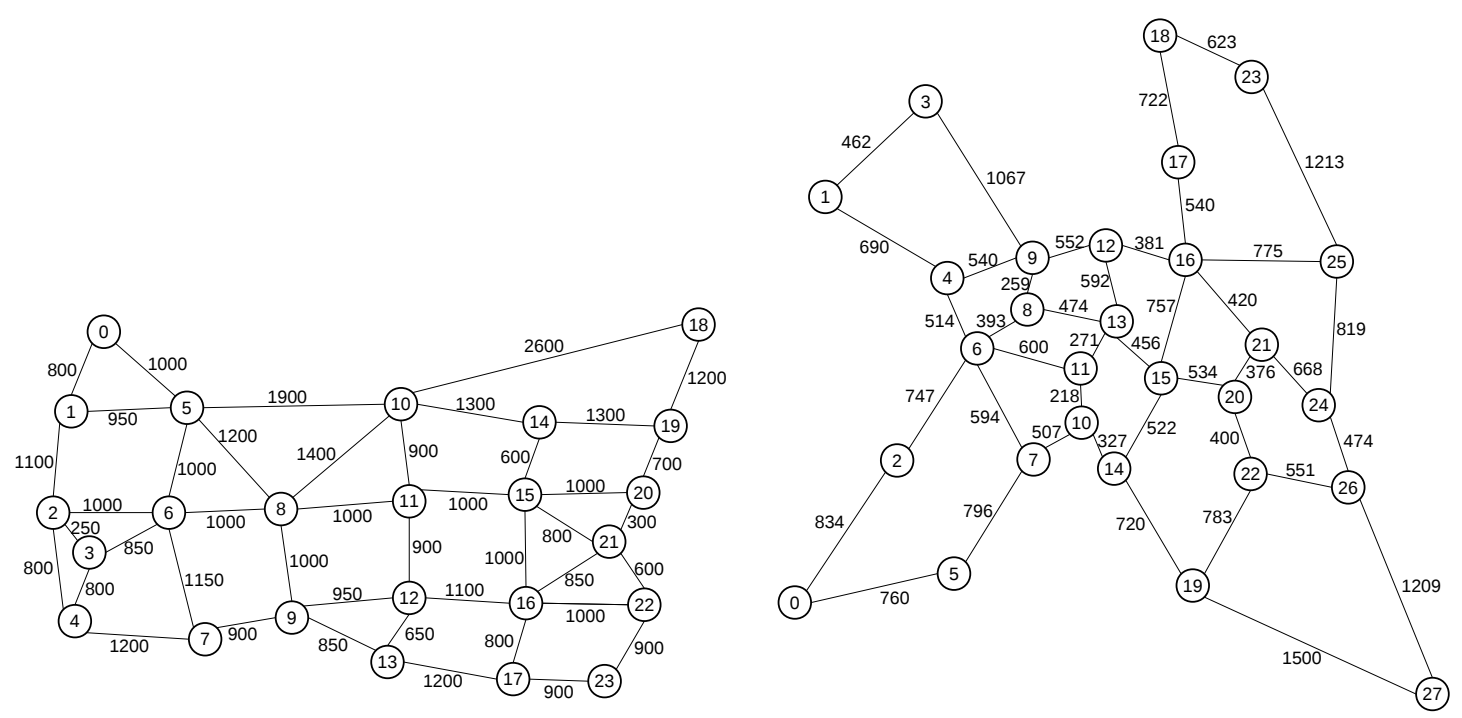

Figura 2. Topologia USANet e PanEuro.

\subsection{Considerações sobre Consumo de Energia}

As hipóteses para mensurar seu gasto energético dos componentes EON (BVT/BV-OXC) está de acordo com [Vizcaíno et al. 2012] e [Ren et al. 2016]. O consumo de energia de um único BVT pode ser calculado de acordo com a Equação 1, onde " $s$ " representa o número de slots utilizados e 133,416(W) representa o consumo energético de cada slot no formato de modulação QPSK. Além disso, um adicional de 68, $3 \%$ é considerado para possíveis sobrecargas mais $91,333 \mathrm{~W}$ de consumo em stand-by para cada BVT.

$$
P C_{B V T}(W)=1,683 *(s * 133,416)+91,333
$$

O consumo energético dos BV-OXCs é dado em função do grau de conexão de cada nó $N$ e do número de canais que o BV-OXC pode adicionar ou remover na rede $(\varepsilon)$. Além disso, são adicionados $150 \mathrm{~W}$ de consumo em stand-by por nó como mostra a Equação 2.

$$
P C_{O X C}(W)=N * 85+\varepsilon \times 100+150
$$

São considerados ainda, mais $30 \mathrm{~W}$ por cada OLA (Optical Line Amplifier) utilizado pelo caminho óptico. Deve ser colocado um OLA para cada enlace físico $(m, n)$ indicado por $A_{m n}$, onde $m$ e $n$ são dois nós da rede. A quantidade de OLAs pode ser calculado de acordo com a Equação 3.

$$
A_{m n}=\left\lceil L_{m n} / S-1\right\rceil+2
$$

onde, $S$ é a distância entre dois OLAs vizinhos, normalmente $S=80 \mathrm{~km}$, e $L_{m n}$ é a distância do enlace $(m, n)$ em km. São somados mais " 2 " amplificadores para as duas extremidades do enlace. Além disso, são considerados mais $140 \mathrm{~W}$ de consumo em standby por par de OLA. Portanto, pode-se calcular o gasto energético por OLA conforme a Equação 4.

$$
P C_{O L A}(W)=A_{m n} *(30+70)
$$




\subsection{Resultados Numéricos}

O BBR (Figura 3) reflete a taxa de banda bloqueada, quanto maior essa taxa, maior é a banda bloqueada. É possível observar que o desempenho do BBR do MinBVT e do MinBV-OXC obtiveram resultados próximos aos melhores algoritmos comparados, em torno de $6 \%$ a $11 \%$ nas cargas mais elevadas. Na topologia USANet, para a maior carga de tráfego, o MinBVT conseguiu um ganho de até $54 \%$ em relação ao MVH-SRLP, esse valor é ainda maior quando comparado aos demais algoritmos. O ganho médio foi de cerca de $15 \%$ em relação ao MVH-SRLP. Para topologia PanEuro, o MinBVT foi ainda melhor, com um ganho médio em torno de $42 \%$ no BBR. Para maior carga de tráfego, o MinBVT conseguiu um ganho de até $52 \%$ quando comparado ao MVH-SRLP. Isso demonstra que a abordagem proposta pelo nosso AG atinge um melhor desempenho BBR que os outros algoritmos baseados em grafo auxiliar. Isso acontece devido à "Camada Óptica" proposta, que proporciona um uso melhor da capacidade dos BVTs encontrando novos arranjos de agregação de tráfego para o problema RSA. Observa-se também que o LBSR possui o pior desempenho de BBR seguido do GGA-MP. Isso ocorre porque o LBSR não utiliza a agregação óptica em sua solução. Por outro lado, embora o GGA-MP utilize agregação óptica, ele é muito restrito na escolha da rota RSA, portanto, em redes mais restritas, como a PanEuro, seu desempenho é pior devido ao uso dos mesmos nós na rota escolhida.
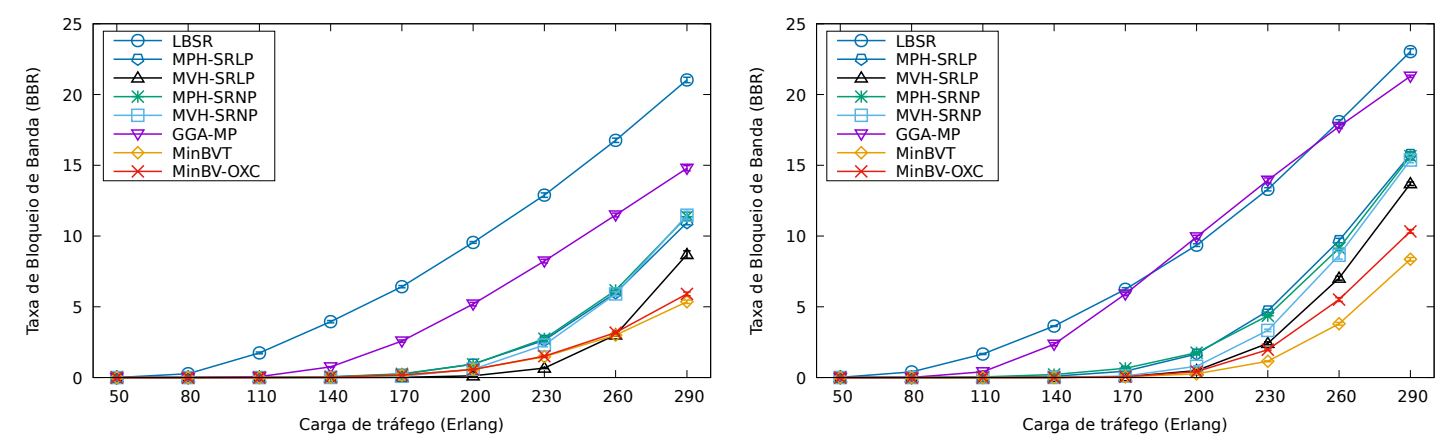

Figura 3. Taxa de bloqueio de banda (BBR). Topologias USANet e PanEuro, respectivamente.

O consumo energético (Figura 4) reflete o gasto de energia de todos os componentes EON em toda a simulação. Nota-se que o MinBVT e o MinBV-OXC proporcionam economias de energia significativas. Ao longo da simulação, o gasto de energia é próximo a $1400 \mathrm{MWh}$ em ambas as topologias, mesmo com o aumento do tráfego. A abordagem proposta oferece ganhos de até $10 \%$ na eficiência energética da rede, em comparação com o MVH-SRLP, que alcança os melhores valores em termos de BBR dentre os algoritmos da literatura. Em comparação com o GGA-MP, os algoritmos propostos mantiveram o mesmo desempenho de energia para as cargas mais baixas. Para maiores cargas, o GGAMP oferece um melhor desempenho energético devido ao maior bloqueio de solicitações de tráfego. Este resultado demonstra que o uso das políticas de redução BVT e BV-OXC associadas ao modelo de grafo auxiliar proposto está em linha com as abordagens Sleep Mode. Portanto, é possível concluir que o esquema proposto envolve necessariamente o uso de menos dispositivos de rede. Os resultados também mostram que os esquemas de reserva de espectro utilizados pela literatura fornecem o uso de mais recursos de rede, causando um gasto maior de energia na rede. 

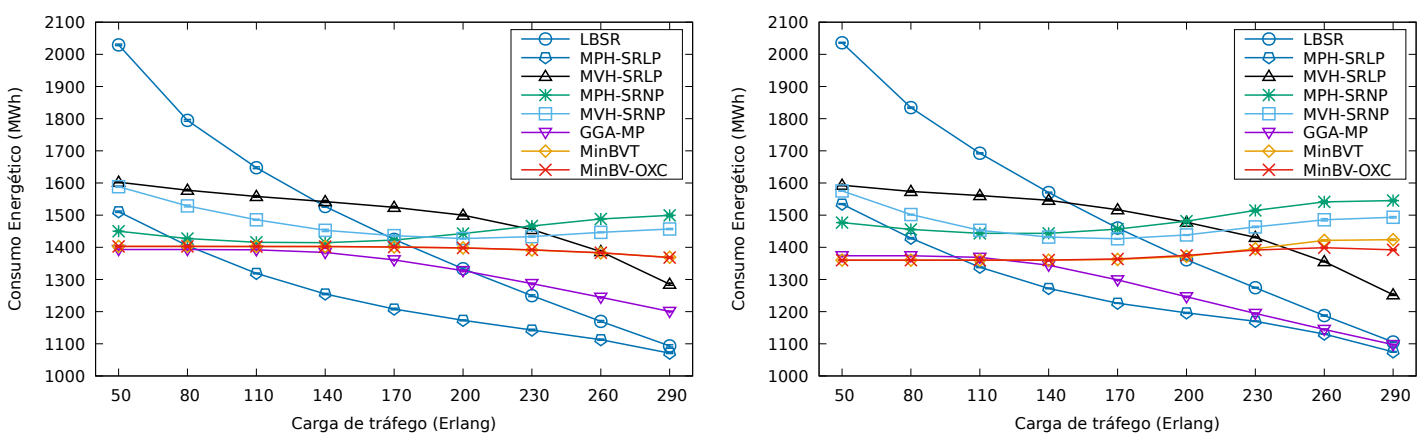

Figura 4. Consumo Energético (MWh). Topologias USANet e PanEuro, respectivamente.

O número de saltos na topologia virtual (Figura 5) indica o número de conversões OEO e processamento elétrico que são utilizados na rede o que impacta na latência total de transmissão. O algoritmo GGA-MP mantém em todas as cargas de tráfego uma média igual a 1, pois este algoritmo é singlehop. Pode-se observar que o MinBVT e o MinBVOXC obtêm o melhor desempenho no número de saltos virtuais, próximo a $1 \mathrm{em}$ ambas as topologias. Isso significa que esses algoritmos fazem menos conversões OEO, portanto, eles utilizam menos BVTs em suas soluções RSA. Observa-se também que o MVH-SRLP aumenta o número de saltos virtuais em cargas de tráfego elevadas, chegando a 2 na topologia PanEuro. Isso demonstra que o AG proposto é capaz de utilizar as técnicas de agregação óptica de forma mais eficiente que o MVH-SRLP, estabelecendo soluções RSA com maior reutilização de BVTs e evidenciando o pior desempenho do MVH-SRLP na eficiência energética.
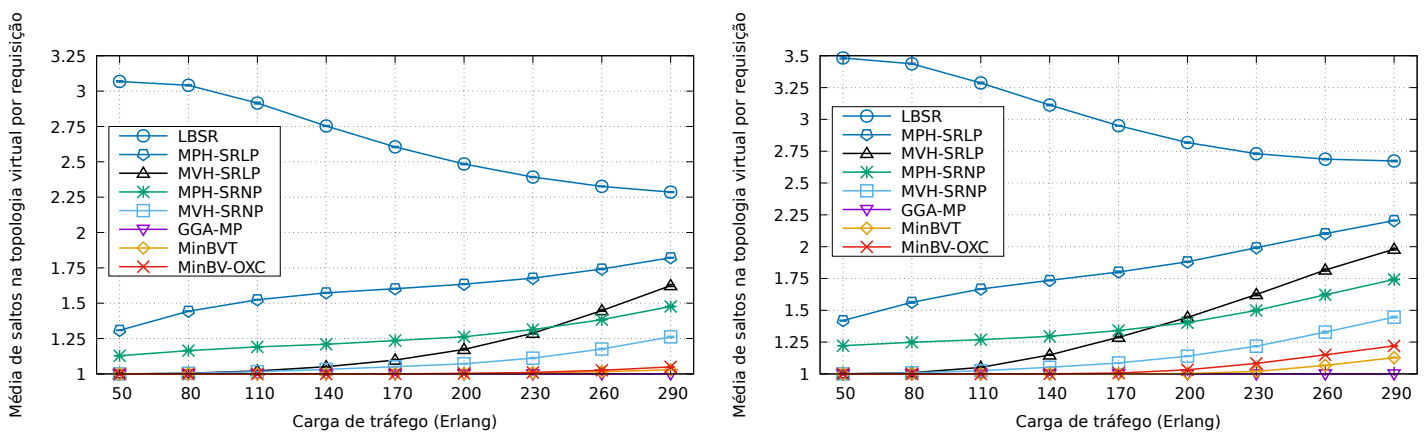

Figura 5. Média de saltos na topologia virtual por requisição. Topologias USANet e PanEuro, respectivamente.

\section{Conclusão}

Este trabalho propõe um modelo de grafo auxiliar inovador para resolver o problema RSA, fornecendo eficiência energética sem sacrificar o desempenho da EON. O novo modelo de gráfico auxiliar proposto amplia as recentes propostas da literatura empregando técnicas de agregação elétrica e óptica utilizando uma menor quantidade de dispositivos conectados na rede. Além disso, são propostas duas políticas para eficiência energética com base na redução do número de BVTs e no número de BV-OXCs utilizados na solução. Para avaliar a solução proposta, foram comparados seis algoritmos da literatura. Os resultados numéricos mostram que a solução proposta é capaz de reduzir em até $10 \%$ o consumo energético, ao mesmo tempo que reduz em até $54 \%$ a taxa de bloqueio de banda em comparação com o estado da arte. 


\section{Referências}

Andrae, A. and Corcoran, P. M. (2013). Emerging trends in electricity consumption for consumer ICT. Technical report, ARAN - Access to Research at NUI Galway.

Cisco (2017). The Zettabyte Era - Trends and Analysis. Cisco Visual Networking Index (VNI) Forecast.

Costa, L. R., de Sousa, L. S., de Oliveira, F. R., da Silva, K. A., Júnior, P. J. S., and Drummond, A. C. (2016). ONS: Optical Network Simulator - WDM/EON. http://comnet.unb.br/br/grupos/get/ons.

Dharmaweera, M. N., Parthiban, R., and Sekercioglu, Y. A. (2015). Toward a power-efficient backbone network: The state of research. IEEE Communications Surveys Tutorials, 17(1):198227.

Heddeghem, W. V., Idzikowski, F., Rouzic, E. L., Mazeas, J. Y., Poignant, H., Salaun, S., Lannoo, B., and Colle, D. (2012). Evaluation of power rating of core network equipment in practical deployments. In 2012 IEEE Online Conference on Green Communications (GreenCom), pages $126-132$.

Idzikowski, F., Chiaraviglio, L., Cianfrani, A., Vizcaíno, J. L., Polverini, M., and Ye, Y. (2016). A survey on energy-aware design and operation of core networks. IEEE Communications Surveys Tutorials, 18(2):1453-1499.

Nag, A., Wang, T., and Mukherjee, B. (2013). Robust design of spectrum-efficient green optical backbone networks. Journal of Lightwave Technology, 31(7):1138-1144.

Ren, R., Hou, W., Guo, L., Liu, Y., Wu, J., and Yang, Y. (2016). Spectrum and energy-efficient survivable routing algorithm in elastic optical network. Optik - International Journal for Light and Electron Optics, 127(20):8795 - 8806.

Tomkos, I., Azodolmolky, S., Sole-Pareta, J., Careglio, D., and Palkopoulou, E. (2014). A tutorial on the flexible optical networking paradigm: State of the art, trends, and research challenges. Proceedings of the IEEE, 102(9):1317-1337.

Vizcaíno, J. L., Ye, Y., and Monroy, I. T. (2012). Energy efficiency analysis for dynamic routing in optical transport networks. In 2012 IEEE International Conference on Communications (ICC), pages 3009-3014.

Wu, Y., Hou, W., Guo, L., Liu, Y., and Sun, Z. (2014). Green grooming in elastic optical networks. In Optical Fiber Communications Conference and Exhibition (OFC), 2014, pages 1-3.

Zhang, J., Ji, Y., Song, M., Zhao, Y., Yu, X., Zhang, J., and Mukherjee, B. (2015). Dynamic traffic grooming in sliceable bandwidth-variable transponder-enabled elastic optical networks. Journal of Lightwave Technology, 33(1):183-191.

Zhang, S., Martel, C., and Mukherjee, B. (2013). Dynamic traffic grooming in elastic optical networks. IEEE Journal on Selected Areas in Communications, 31(1):4-12.

Zhu, H., Zang, H., Zhu, K., and Mukherjee, B. (2003). A novel generic graph model for traffic grooming in heterogeneous WDM mesh networks. IEEE/ACM Transactions on Networking, 11(2):285-299. 